\title{
DEVELOPMENT OF TECHNIQUES FOR CLEARANCE OF SPENT SEALED NUCLEAR MEDICINE CALIBRATION SOURCES
}

\author{
Dimitrios Mavrikis $^{1,2^{*}}$, Alexandra loannidou ${ }^{2}$, Anastasia Savidou ${ }^{1}$
}

${ }^{1}$ National Centre for Scientific Research “Demokritos”, Institute of Nuclear \& Radiological Sciences \& Technology, Energy and Safety, Radioactive Waste \& Material Laboratory

2Aristotle University of Thessaloniki, Physics Department, Nuclear Physics and Elementary Particle Physics Division

\begin{abstract}
The present work concerns the development of techniques which can be used to verify clearance of Ge-68/ Ga-68 and Co-57 sealed radioactive sources. These sources are used for the calibration of various nuclear medicine systems like Gamma camera and PET imaging. There are several types of such sources of different characteristics and geometry i.e. linear, area, volume, while after their useful life these sealed sources need to be kept in control for decay till meeting the clearance criteria. The techniques aim to determine the activity of the sources after storage for appropriate time span until the clearance criteria are met and are based on Monte Carlo simulation using the MCNPX code for evaluation of the $3 \times 3 \mathrm{NaI}$ (Tl) scintillator detector efficiency. A preliminary work was done for two types of sealed radioactive sources: i) a line source containing Ge-68/Ga-68, ii) a flood source containing Co-57. However, the determined activities were underestimated by $13.6 \%$ for Co-57 flood source and $26.9 \%$ for Ge-68 line source, compared to the source's nominal activities. In the present study, the accuracy of the previously developed techniques has been improved by detailed detector characterization.
\end{abstract}

Keywords: Clearance, gamma-ray spectrometry, MCNPX, NaI (Tl), simulations, sealed sources

\section{INTRODUCTION}

Nowadays, there is a variety of sealed radioactive sources, such as Co-57 and Ge-68/Ga-68, which are used for the precise calibration of nuclear medicine systems. After their useful life, these sources need to be handled and kept into storage until they meet the general clearance criterion. In some cases, the sources certificates with their characteristics and nominal activity have been lost, so their current activity cannot be estimated. For the experimental determination of the source activity, the detector efficiency should be evaluated for the specific source-detector geometry. In case of complex geometries, a common approach to perform the efficiency calibration is Monte Carlo simulation techniques. In past work [1], a semiempirical method was developed based on Monte Carlo simulation by utilizing the MCNPX code for evaluation of the 3'x3' $\mathrm{NaI}(\mathrm{Tl})$ scintillator efficiency for specific source-detector geometries. In this study, a more accurate determination of the $\mathrm{NaI}(\mathrm{Tl})$ crystal description was made, aiming on the more accurate activity estimation of the sources.

Furthermore, gamma-ray spectra were taken for different acquisition time by a NaI(Tl) detector for two types of sealed radioactive sources: 1) a flood source containing Co-57; 2) a line source containing Ge-68/Ga-68. For these source-detector geometries and the specific gamma ray energies, simulations were performed with the MCNPX code. The MCNPX models were validated by the certified activities of radiation sources of these types.

\section{MATERIALS AND METHODS}

\subsection{Detection system and calibration source}

The gamma spectrometry system consists of the following main parts:

- Detector unit: A Bicron Monoline scintillation detector $\mathrm{NaI}(\mathrm{Tl})$ (Model $3 \mathrm{M} 3 / 3$ ) with a $3^{\prime} \times 3^{\prime}$ crystal in a thick aluminum housing, covered with a white reflector, including a photomultiplier tube, an internal magnetic/light shield and a 14-pin connector

- Electronics and acquisition unit: a digital signal processing unit (Osprey Digital Tube Base MCA) and a high voltage supply system (850 V)

- GenieTM 2000 spectroscopy software for spectrum acquisition [2].

\footnotetext{
* d.mavrikis@ipta.demokritos.gr
} 
The experimental data were obtained from a multiple gamma ray volume certified source of $1.5 \mathrm{~g} / \mathrm{cm}^{3}$ epoxy material density. The main features of the source are shown in Table 1.

Table 1. Characteristics of

the multiple gamma-ray volume source

\begin{tabular}{|c|c|c|c|c|}
\hline $\begin{array}{c}\text { Multiple } \\
\text { volume } \\
\text { source } \\
\text { density } \\
\left(\mathrm{g} / \mathrm{cm}^{3}\right)\end{array}$ & Isotopes & $\mathrm{T}^{1 / 2}(\mathrm{~d})$ & $\begin{array}{c}\text { Reference } \\
\text { Activity } \\
(\mathrm{kBq})\end{array}$ & $\begin{array}{c}\text { Date of } \\
\text { Calibration }\end{array}$ \\
\hline & ${ }^{241} \mathrm{Am}$, & 157753, & $1.26 \pm 3.6 \%$ & \\
$1.5 \pm 0.1 \mathrm{~g} / \mathrm{cc}$ & ${ }^{137 \mathrm{Cs},}$ & 11001, & $2.66 \pm 2.9 \%$ & $1 / 3 / 2007$ \\
& ${ }^{60} \mathrm{Co}$, & 1923.55, & $3.32 \pm 2.9 \%$ & \\
& ${ }^{210} \mathrm{~Pb}$ & 7665 & $12.6 \pm 2.9 \%$ & \\
\hline
\end{tabular}

\subsection{Experimental Efficiency Calibration}

The experimental full - energy peak efficiency Eexp is the ratio of the number of counts $\mathrm{N}$ detected in a peak to the number of photons emitted by the source [3]. The formula is $\operatorname{Eexp}=\mathrm{N} /\left(\mathrm{A}^{*} \mathrm{t}^{*} \mathrm{P} \gamma\right)$ where $\mathrm{N}$ are the number of counts detected, $\mathrm{A}$ is the source activity, $t$ is the acquisition time and $\mathrm{P} \gamma$ is the probability of emission of the particular gamma ray being measured (peak energy abundance).

The main factors that may affect the calibration curve are different source to detector distance, different shape of source, absorption within the source, random summing at high counting rate, true coincidence summing at close geometry [3]. The dead time during the measurements was less than $2 \%$ and the true coincidence summing for the Co-6o photon peaks was corrected.

The experimental data were obtained from the multiple gamma-ray volume source of $1.5 \mathrm{~g} / \mathrm{cm}^{3}$ density epoxy material (Table 1). Experimental

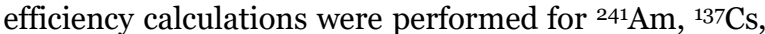
${ }^{60} \mathrm{Co}$ and ${ }^{210} \mathrm{~Pb}$ with the volume sources adapted on an acetal holder (Fig. 1) which was positioned in a vertical direction along the axis of symmetry. The acquisition time was $1 \mathrm{~h}$ and $40 \mathrm{~min}$.

\subsection{Monte Carlo Simulations for Efficiency Calibration}

MCNPX simulations were performed in an energy range of $45-1332 \mathrm{keV}$ including the energies used in the experimental measurement for the multiple gamma-ray large volume sources of $1.5 \mathrm{~g} / \mathrm{cm}^{3}$ epoxy material density. For the efficiency estimation "F8" tally is used which describes the energy distribution of pulses created in a detector by radiation. The visualization of $\mathrm{NaI}$ crystal, acetal holder and the active volume source is shown in Fig. 1.

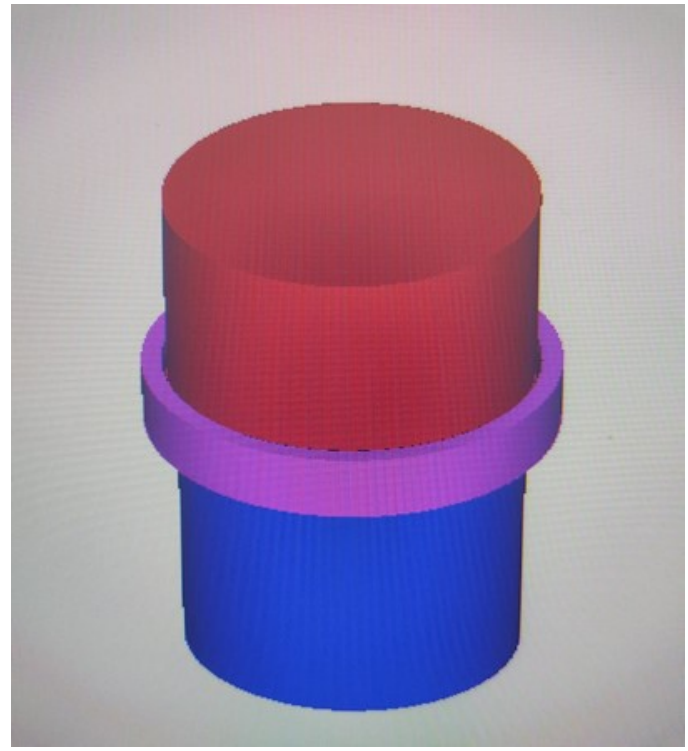

Figure 1. MCNP visualization: the detector in blue, the volume source in red and the acetal holder in purple

\subsection{Radioactive Sources Measurements}

Gamma-ray spectra were taken for different acquisition time by the $3^{\prime} \times 3^{\prime} \mathrm{NaI}(\mathrm{Tl})$ detector for two types of sealed radioactive sources with $15 \%$ uncertainty of the certified activities:

i. a line source containing Ge-68/Ga-68 (Fig.2, left)

ii. a flood source containing Co-57 (Fig.2, right)
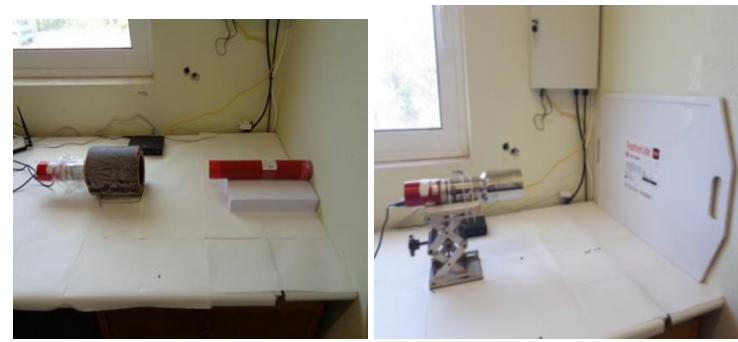

Figure 2. Experimental setup for Ge-68/Ga-68 line source (left) and Co-57 flood source (right)

Gamma-ray spectra of the two sources were taken for several acquisition times, 7 hours, 1 hour and 15 minutes for the Ge-68/Ga-68 line source and for 7 hours, 2 hours and 15 minutes for the Co-57 flood source. Ge-68 decays by electron capture to Ga-68 which is mainly a positron emitter $(89.1 \%)$ leading to $\mathrm{Zn}-68$. Then, the excited state of the daughter nucleus Zn-68 decays by a gamma emission of $1077 \mathrm{keV}$ energy (2.93\%) [4] (Fig. 3). Co-57 decays by electron capture to the excited state of Fe-57 and subsequently $\gamma$ photons with energies $122 \mathrm{keV}$ (85.6\%), $136 \mathrm{keV}$ (10.68\%) and $692.03 \mathrm{keV}$ (0.157\%) are emitted leading to the ground state of $\mathrm{Fe}-57$ [4]. The characteristic gamma ray peaks in the resulted spectra were analyzed with the software package SPECTRW [5]. For the Co-57 source, deconvolution of the peak energies $122 \mathrm{keV}$ and $136 \mathrm{keV}$ was carried out by using this software package [5] (Fig. 3). 
For the source-detector geometries and the specific gamma ray energies, simulations were performed by the MCNPX code for evaluation of the NaI(Tl) detector efficiencies. The detector main axis of symmetry was placed perpendicular to the sources flat surfaces. The sources were placed at such distances from the detector, in order acceptable dead time (less than $5 \%$ ) to be achieved (Fig. 4).
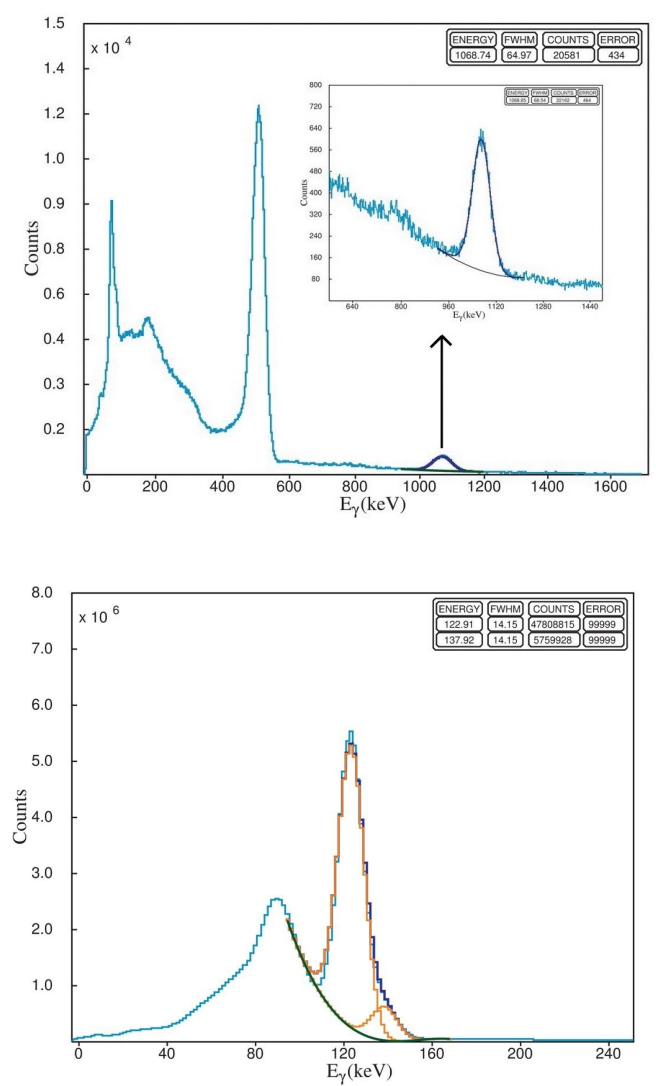

Figure 3. Ge-68/Ga-68 line source spectrum, 1h exposure (up) and Co-57 flood source spectrum, $2 \mathrm{~h}$ exposure (down)
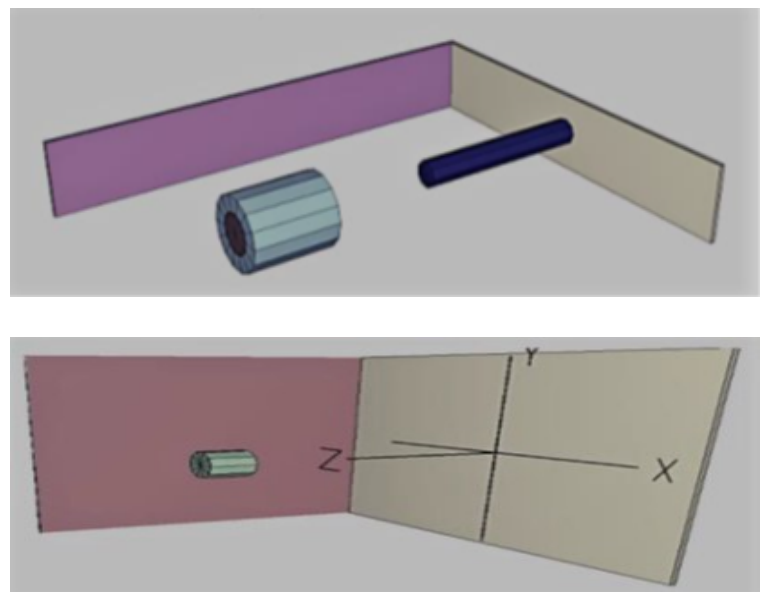

Figure 4. Ge-68/Ga-68 line source MCNPX visualization (up) and Co-57 flood source MCNPX visualization (down)
Specifically, the Ge-68/Ga-68 line source (ceramic, height $16.3 \mathrm{~cm}$, radius $0.07 \mathrm{~cm}$ ) was measured with the cylindrical $\mathrm{Pb}$ shielding (height $37 \mathrm{~cm}$, radius $2.85 \mathrm{~cm}$, thickness $1.52 \mathrm{~cm}$ ) for radiation protection purposes. $\mathrm{Pb}$ cylindrical shielding was placed around the detector to reduce the background radiation (height $18 \mathrm{~cm}$, radius $7.5 \mathrm{~cm}$, thickness $3.3 \mathrm{~cm}$ ). The shielding of the source was placed at the distance of $25 \mathrm{~cm}$ from the detector (Fig. 2 \& 4). The Co-57 flood source (length $61 \mathrm{~cm}$ and height $41.9 \mathrm{~cm}$ ) was placed at the distance of $47 \mathrm{~cm}$ from the detector (Fig. $2 \& 4$ ).

\section{RESULTS AND DISCUSSION}

For the detector efficiency calibration, MCNPX simulations were performed in an energy range of 45 - $1332 \mathrm{keV}$ including the energies used in the experimental calculations for the $1.5 \mathrm{~g} / \mathrm{cm}^{3}$ multiple gamma - ray volume source. The theoretical efficiency curve, determined by MCNPX simulations, for a $3 \times 3 \mathrm{NaI}(\mathrm{Tl})$ detector (initial curve) was not in a good agreement compared with the experimental data. In order to correct the theoretical efficiency curve, two cases were examined for the crystal description:

i) an air gap between $\mathrm{NaI}$ Crystal and aluminium housing at the upper surface of the detector

ii) an inactive NaI layer on the external surface of the NaI crystal

Multiple MCNPX simulations were done in each case. For the first option the air gap between $\mathrm{NaI}$ crystal and $\mathrm{Al}$ housing was increased by a step of $0.5 \mathrm{~mm}$, from omm up to $2 \mathrm{~mm}$. In the second option the inactive NaI layer was increased by a step of $0.5 \mathrm{~mm}$, from omm up to $2 \mathrm{~mm}$. The option of a $0.5 \mathrm{~mm}$ thick inactive $\mathrm{NaI}$ layer on the external surface of the crystal was in the best agreement with the experimental data. The MCNPX simulation curves for efficiency calibration in case of $2 \mathrm{~mm}$ air gap between NaI crystal and aluminum housing and $0.5 \mathrm{~mm}$ inactive NaI layer on the external surface of the crystal are shown in Fig. 5 \& Fig. 6 respectively.

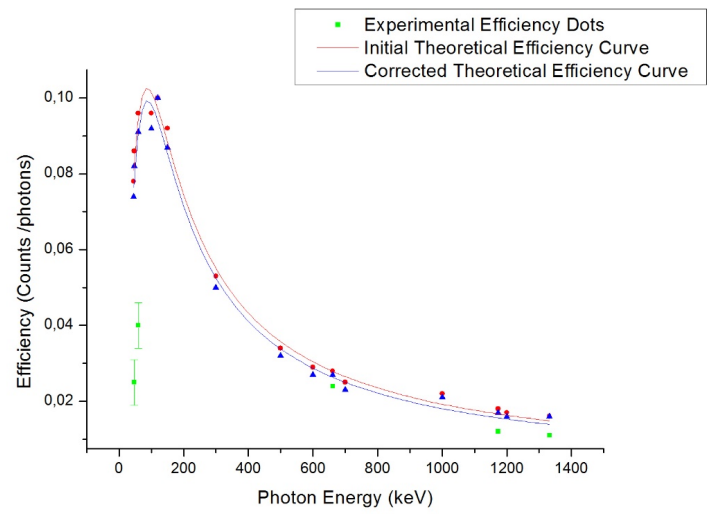

Figure 5. Correction of theoretical efficiency curve. First option: air gap between $\mathrm{NaI}$ crystal and $\mathrm{Al}$ housing 


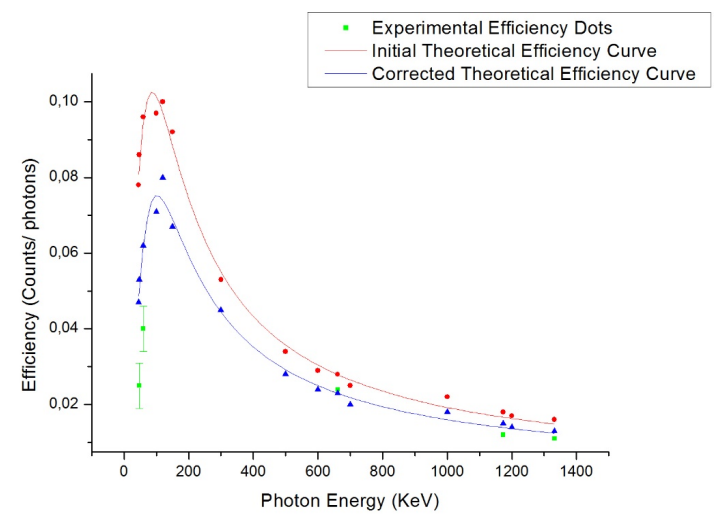

Figure 6. Correction of theoretical efficiency curve. Second option: inactive NaI layer in the external surface of the crystal

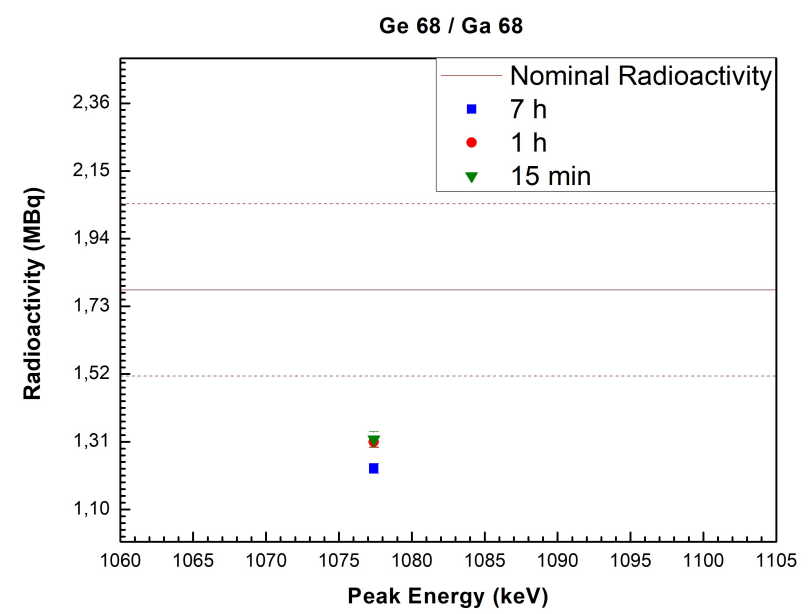

In low energies, the experimental efficiency for $\mathrm{Am}-241$ and $\mathrm{Pb}-210$ is much lower than the expected value. Possibly, this is due to the significant measurement error.

The results of the study show that the acquisition time of two hours provided adequate statistics for the activity determination of the flood source Co- 57 . According to the previous work [1], the determined activity had a deviation of $13.6 \%$ from the source nominal activity. Also, one hour measurement was adequate for the activity determination of the line source Ge-68, with a deviation of $26.9 \%$ from the nominal activity (Fig. 7). After the better description of the $\mathrm{NaI}$ crystal, the new activities were determined (Fig. 8) and the deviation from each source nominal activity was reduced to $6.6 \%$ for Co-57 and to $14.6 \%$ for Ge-68.

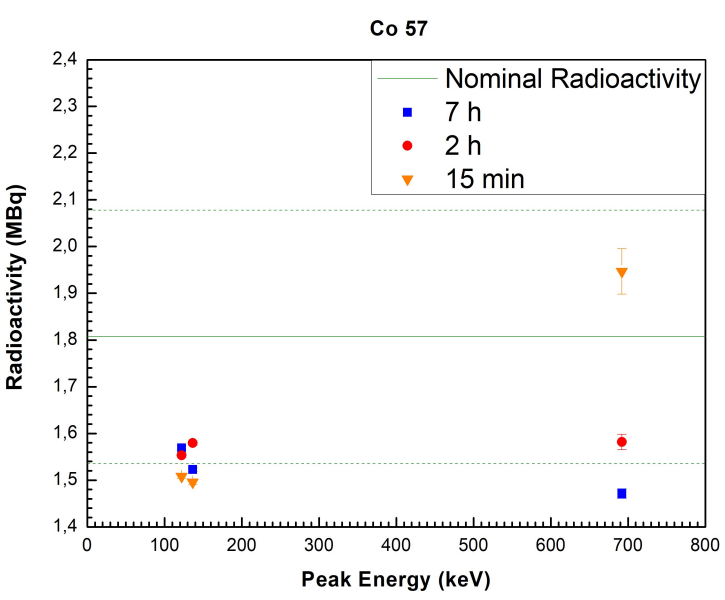

Figure 7. Previously determined activities for different acquisition time compared with the nominal activity
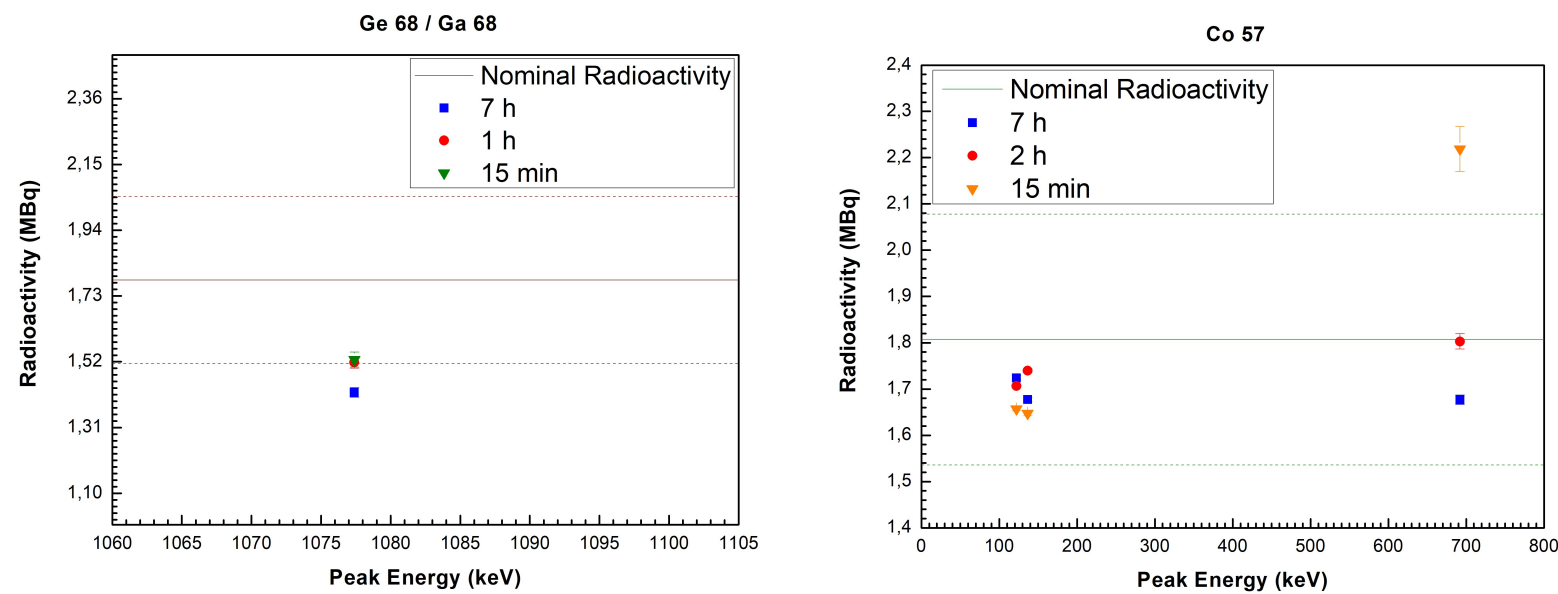

Figure 8. Corrected activities for different acquisition time compared with the nominal activity 
The general clearance criterion for $\mathrm{Co}-57$ is $1 \mathrm{~Bq} / \mathrm{g}$ and for Ge-68/Ga-68 is $10 \mathrm{~Bq} / \mathrm{g}[6,7]$. The specific activities are given in Table 2.

Table 2. Corrected and initial determined activities for different acquisition time

\begin{tabular}{cccc}
\hline Energy & Duration & $\begin{array}{c}\text { Corrected Activity } \\
\text { (MBq) }\end{array}$ & $\begin{array}{c}\text { Initial Activity } \\
\text { (MBq) }\end{array}$ \\
\hline & \multicolumn{3}{c}{ Co-57 } \\
\hline 122.06 & $7 \mathrm{~h}$ & $1.723 \pm 0.005$ & $1.569 \pm 0.004$ \\
136.47 & $7 \mathrm{~h}$ & $1.677 \pm 0.004$ & $1.523 \pm 0.004$ \\
692.03 & $7 \mathrm{~h}$ & $1.676 \pm 0.011$ & $1.471 \pm 0.010$ \\
122.06 & $2 \mathrm{~h}$ & $1.706 \pm 0.004$ & $1.553 \pm 0.004$ \\
136.47 & $2 \mathrm{~h}$ & $1.739 \pm 0.005$ & $1.579 \pm 0.005$ \\
692.03 & $2 \mathrm{~h}$ & $1.802 \pm 0.018$ & $1.582 \pm 0.016$ \\
122.06 & $15 \mathrm{~min}$ & $1.656 \pm 0.004$ & $1.508 \pm 0.004$ \\
136.47 & $15 \mathrm{~min}$ & $1.647 \pm 0.005$ & $1.496 \pm 0.005$ \\
692.03 & $15 \mathrm{~min}$ & $2.218 \pm 0.055$ & $1.946 \pm 0.048$ \\
\hline & & Ge-68/Ga-68 & \\
1077.4 & $7 \mathrm{~h}$ & $1.421 \pm 0.017$ & $1.227 \pm 0.015$ \\
1077.4 & $1 \mathrm{~h}$ & $1.517 \pm 0.020$ & $1.310 \pm 0.018$ \\
1077.4 & $15 \mathrm{~min}$ & $1.526 \pm 0.027$ & $1.319 \pm 0.024$
\end{tabular}

\section{CONCLUSION}

In this study, an effect of accurate description of the $\mathrm{NaI}(\mathrm{Tl})$ crystal on the accuracy of detector efficiency calibration is presented. Regarding the efficiency calibration, a semi-empirical method was used based on a combination of experimental gamma spectrometry measurements and MCNPX simulations. Simulated and experimentally calculated efficiencies were compared and the results showed that a good agreement was obtained when an inactive $2 \mathrm{~mm} \mathrm{NaI}$ layer on the external surface of the NaI crystal was considered. By using the corrected efficiency curve, the accuracy of determination of the Co-57 and Ge-68/Ga68 radioactive sources activities was increased.

\section{REFERENCES}

1. T. Siarafera et al., "Development of techniques based on Monte Carlo simulations for clearance of Co-57 and Ge-68/Ga-68 sealed radioactive sources," in Proc. 27th
Annu. Symp. Hellenic Nuclear Physics Society (HNPS 2018), Athens, Greece, 2018.

DOI: $10.12681 / \mathrm{hnps} .1819$

2. GenieTM 2000 Gamma Analysis Spectroscopy Software, Canberra Industries, Inc., Meriden (CT), USA.

3. G. Gilmore, Practical Gamma-Ray Spectrometry, 2nd ed., Chichester, England: Wiley \& Sons, 2008. Retrieved from: http://library.lol/main/1903C10635634147A56oB5F10 7 EoD42E

Retrieved on: Jan. 5, 2021

4. R. B. Firestone, Table of Isotopes, 8th ed., Hoboken (NJ), USA: Wiley Interscience, 1996.

Retrieved from:

http://library.lol/main/ACC5FoC59EA8E3E1FE349EF EB1487E01

Retrieved on: Nov. 20, 2020

5. L. Done, M-R. Ioan, "Minimum Detectable Activity in gamma spectrometry and its use in low level activity measurements," Appl. Rad. Isot., vol. 114, pp. 28 - 32, Aug. 2016.

DOI: 10.1016/j.apradiso.2016.05.004

6. C. A. Kalfas, M. Axiotis, C. Tsabaris, "SPECTRW: A software package for nuclear and atomic spectroscopy," Nucl. Instrum. Methods Phys. Res. A, vol. 830, pp. $265-274$, Sep. 2016.

DOI: 10.1016/j.nima.2016.05.098

7. EФHMEPI $\Sigma$ TH $\Sigma$ KYBEPIH $\Sigma$ E $\Omega \Sigma$ TH $\Sigma$ E $\Lambda \Lambda H N I K H \Sigma$

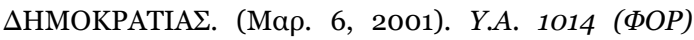

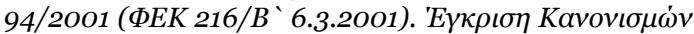
Актіvолробтабіаs.

(Gazette of the government of the Hellenic Republic. $\begin{array}{llllll}\text { (Mar. 6, 2001). WILL. } 1014 & \text { (FOR) 94/2001 }\end{array}$ (Government Gazette 216 / B 6.3.2001). Approval of Radiation Protection Regulations.)

Retrieved from:

https://www.elinyae.gr/sites/default/files/201907/b216 01.1127808638026.pdf

Retrieved on: Dec. 22, 2020

8. Der Schweizerische Bundesrat. (Apr. 26, 2017). Strahlenschutzverordnung (StSV) 814.501, Anhang 2 (Art. 2 Abs. 1 Bst.j, l und $m$ sowie 194 Abs. 3.)

(The Swiss Federal Council. (Apr. 26, 2017). Radiological Protection Ordinance (RPO) 814.501, Appendix 2 (Art. 2 Paragraph 1 Letters $j, l$ and $m$ as well as 194 Paragraph 3.)

Retrieved from:

https://www.fedlex.admin.ch/eli/cc/2017/502/de Retrieved on: Jan. 22, 2021 